\title{
TENDENCIAS DEL MARKETING DEL SIGLO XXI APLICADAS AL EMPRENDIMIENTO SOCIAL
}

\section{TRENDS IN MARKETING OF THE XXI CENTURY APPLIED TO SOCIAL ENTREPRENEURSHIP}

\author{
Contrera-Zambrano Ángel ${ }^{1 *}$; Velásquez-Reyes Gabriel²; Becerra-Ávila Mauricio ${ }^{3}$ \\ ${ }^{1}$ Director de Zoom-marketing. Manta, Ecuador. \\ ${ }^{2}$ Director legal de La Casa del Abogado. Manta, Ecuador. \\ ${ }^{3}$ Docente de la Universidad Laica Eloy Alfaro de Manabí, ULEAM. Manta, Ecuador.
}

*Correo: zomm-mar-19@hotmail.com

\begin{abstract}
Resumen
El marketing aporta valiosas estrategias en el desarrollo de proyectos de emprendimiento que permiten posicionar este tipo de negocios en el mercado. Por su parte, el emprendimiento social requiere de estrategias mercadotécnicas de carácter no lucrativo, que si bien pretenden diseñar y poner en marcha programas y proyectos sociales, no se aíslan de los conceptos básicos del marketing asociados con la permanente planificación de producto, precio, comunicación, distribución e investigación. En este trabajo se busca revisar la literatura científica referente a la estructura del marketing en el siglo XXI y su incidencia en el desarrollo del emprendimiento. EI $90 \%$ de los emprendimientos no llega a los 3 años de vida, por lo tanto, a través de la investigación analítica, prospección estratégica y la inteligencia de negocios el marketing estratégico cuenta con herramientas especializadas y metodologías de vanguardia para generar la visibilidad deseada tanto en un proyecto de emprendimiento comercial como social.
\end{abstract}

Palabras clave: marketing, tendencias, sociedad, proyectos.

\begin{abstract}
Marketing provides valuable strategies in the development of entrepreneurship projects that allow positioning this type of business in the market. For its part, social entrepreneurship requires nonprofit marketing strategies, which although they intend to design and implement social programs and projects, are not isolated from the basic concepts of marketing associated with the permanent planning of product, price, communication, distribution and research. This paper seeks to review the scientific literature regarding the structure of marketing in the 21 st century and its impact on the development of entrepreneurship. $90 \%$ of the ventures do not reach 3 years of life, therefore, through analytical research, strategic prospecting and business intelligence, strategic marketing has specialized tools and cutting-edge methodologies to generate the desired visibility both in a commercial and social entrepreneurship project.
\end{abstract}

Keywords: marketing, trends, society, projects.

Información del manuscrito:

Fecha de recepción: 10 de septiembre de 2019

Fecha de aceptación: 18 de diciembre de 2019

Fecha de publicación: 10 de enero de 2020 


\section{Introducción}

El emprendimiento del siglo XXI opera en un entorno caracterizado por la globalización. En este aspecto, las pequeñas y medianas empresas pueden ser motores de crecimiento para las innovaciones del mercado de productos y el desarrollo económico de las naciones, el auge de las PYMES internacionales es una tendencia importante. Sin embargo, debido a su tamaño corporativo, la mayoría de las PYME carecen de las capacidades, el poder de mercado y otros recursos con los que cuenta la empresa tradicional (Santos, 2012).

El éxito de los emprendimientos en la globalización depende en gran medida de la formulación e implementación de estrategias. La estrategia refleja las respuestas a corto y largo plazo frente a los desafíos y oportunidades que representa el entorno empresarial. Las compañías ponen en marcha estrategias para atraer clientes $y$ lidiar de manera efectiva con una gran cantidad de preocupaciones. La estrategia de marketing puede apoyarse cuando la gerencia tiene una orientación empresarial (Knight, 2000).
La respuesta de la globalización define las medidas que las empresas toman para responder a las oportunidades y amenazas provocadas por la globalización. Corresponden a la gestión para modificar tácticas de negocios, actividades de marketing y comportamientos del sector externo en general. En los mercados globales, debido a que la empresa enfrenta diversas necesidades de los consumidores y ofertas de los competidores, así como diversas condiciones económicas y tecnológicas, responder a la globalización puede ser particularmente importante para el éxito de las PYME. De hecho, los competidores extranjeros con frecuencia cuentan con el apoyo de los gobiernos locales, utilizan estrategias no anticipadas y se encuentran distribuidos en muchos mercados nacionales. El principal desafío que enfrentan las empresas es crear valor para los compradores de la manera más eficiente posible (Harding, 2006). Las empresas que son altamente receptivas bajo la globalización están mejor posicionadas para realizar esta tarea y deben, en relación con las empresas estáticas que no 
responden, lograr un mayor éxito en los mercados extranjeros (Knight, 2000).

Por otra parte, el marketing estratégico se ha constituido en una herramienta cada vez más empleada en proyectos de emprendimiento, debido a que otorgan visibilidad y una proyección estratégica que con el tiempo permite posicionar el negocio. Además, el emprendimiento social ha ganado mucho espacio en la estructura actual del marketing, puesto que surgen las clasificaciones no lucrativas y mixtas (Dacin et al., 2010). Santos (2012) sugiere que el emprendimiento social es la búsqueda de soluciones sostenibles a problemas desatendidos con externalidades positivas.

En este trabajo se busca revisar la literatura científica referente a la estructura del marketing en el siglo XXI y su incidencia en el desarrollo del emprendimiento.

\section{Estructura del marketing del siglo XXI}

En función de lo mencionado anteriormente, en el siglo anterior ya se habían introducido los elementos que componen la estructura actual del marketing, por lo tanto, ya se hacía referencia a términos como marketing lucrativo, no lucrativo y mixto. La Universidad de Ohio introdujo un debate académico que contribuyó con investigaciones científicas que aportaron al desarrollo de esta disciplina. La base teórica que condujo a dicha estructura se cimienta en la conclusión de trabajo de Lazer (1969): "el marketing tiene dimensión social, por lo tanto, no solo debe tratarse como una tecnología al servicio de la organización empresarial".

El alcance del marketing se amplía en una secuencia vertical y horizontal. En la forma vertical se posiciona la responsabilidad social del marketing, con una estructura jerárquica significativa (Kotler \& Zaltman, 1997).

Por su parte, la secuencia horizontal implica la expansión del marketing desde las organizaciones con objetivos lucrativos hacia las no lucrativas, posicionando de esta forma, el marketing social. Instituciones de carácter social, político, cultural, religioso ofrecen servicios y productos a un segmento importante de la población 
empleando herramientas mercadotécnicas que les permitan dirigirse y posicionarse entre los consumidores; por ende, se vuelve imperiosa la extensión del concepto de esta disciplina hacia las instituciones sin fines de lucro (Kotler \& Levy, 1969).

Tal como sostienen Kotler \& Zaltman (1971, p. 5), "el marketing social se refiere al diseño, puesta en marcha y evaluación de programas desarrollados en aras de influir en la aceptación de proyectos sociales y considerando permanentemente la planificación de producto, precio, comunicación, distribución, e investigación de marketing".

\subsection{Dicotomías del marketing}

El marketing puede estratificarse en tres dicotomías según lo que propone Hunt (1976):

a) Dicotomía sector lucrativo/no lucrativo, permite clasificar a las organizaciones en dependencia de sus objetivos. El primero apunta hacia la obtención de beneficios económicos y el segundo sin afán de lucro, como por ejemplo: Organizaciones no gubernamentales (ONGs) y asociaciones. b) Dicotomía macro/micro, permite diferenciar en función de la escala de las actividades y/o competencias del sector. A nivel macro se consideran las actividades de un conjunto de empresas e instituciones agregadas, así como un grupo de consumidores, en concordancia con la relación causaefecto en el crecimiento del entorno social y económico. El nivel micro, enfoca su campo de acción en las actividades de unidades individuales, tales como: empresas e individuos, con el objetivo de evaluar el desarrollo de los procesos de la institución.

c) Dicotomía positivo/ normativo. El alcance del nivel positivo caracteriza los componentes del objeto de estudio, mientras que el nivel normativo prioriza una prescripción de las actividades que deben desarrollarse para lograr los objetivos. En la industria de producción de artefactos eléctricos, un estudio positivo se basaría en la descripción de las cualidades de los elementos que se ofertan en el mercado; sin embargo, un estudio normativo propondría los precios a los que deben venderse o los 
potenciales clientes o los canales de distribución de los productos.

\subsection{Sectores lucrativos vs no lucrativos}

La investigación de Hunt (1976) contiene elementos que aportan en la comparación de los sectores del marketing. En este contexto, los sectores lucrativos y no lucrativos presentan las siguientes diferencias:

En las instituciones sin fines de lucro, a nivel micro y en un análisis positivo, se evalúa la adquisición de bienes públicos por los consumidores. Por su parte, bajo un análisis normativo, el problema se define como la toma decisiones referentes al precio, consumo, distribución, entre otros.

En el sector no lucrativo, a nivel macro y bajo una perspectiva positiva los problemas se centran en el modelo institucional de los bienes públicos; mientras que bajo un enfoque normativo se plantea si la sociedad debe permitir a los políticos ofertarse como un producto.

Las instituciones con fines de lucro, a escala micro en función del nivel positivo deben resolver problemáticas como el comportamiento del consumidor individual, así como la determinación de precios. No obstante, a nivel normativo, se debe controlar los esfuerzos del marketing y gestionar la venta al detalle.

Las empresas a escala macro evaluadas con un alcance positivo deben determinar eficiencia del sistema y aspectos legales del marketing. A diferencia del régimen normativo, que se enfoca en la búsqueda de mecanismos para aumentar la eficiencia del marketing.

\section{Marketing estratégico en proyectos de emprendimiento}

El $90 \%$ de los emprendimientos no llegan a los 3 años de vida y el 95\% no pasa de los 4 años. A través de la investigación analítica, prospección estratégica y la inteligencia de negocios el marketing estratégico cuenta con herramientas especializadas y metodologías de vanguardia para generar la visibilidad, el conocimiento y la plataforma de pensamiento de prospección estratégica para darle viabilidad en el tiempo al emprendimiento (Chang et al., 2015).

Según Cravens (2006) el objetivo es evaluar la importancia del marketing 
estratégico para potenciar la competitividad y viabilidad en el tiempo del negocio más allá del promedio de vida de los emprendimientos.

La mejor manera de conocer a los clientes es la segmentación. Al segmentar al público objetivo, se gestionará el uso de los recursos disponibles para plantear la estrategia óptima para llegar a los clientes (Morris et al., 2002).

La segmentación consiste en dividir el mercado en grupos que tengan las mismas características y necesidades para atenderlos de forma específica y diferenciada (Kumar et al., 2016).

Los proyectos empresariales enfocados en emprendimientos deben operar en un entorno que presenta mayores riesgos, una menor capacidad para pronosticar, una empresa fluida, los límites de la industria, una mentalidad de gestión que debe desaprender los principios de administración tradicionales y adquirir nuevas metodologías estructurales que permitan no solamente el cambio, sino que también ayuden a crearlo.
Algunas

innovaciones sorprendentes del emprendimiento social provienen de países en desarrollo e implican el despliegue de nuevos modelos de negocio que abordan las necesidades humanas básicas (Seelos \& Mair, 2005).

\section{Consideraciones finales}

Kotler (2001) señala que el término marketing empresarial (ME) se ha usado de varias maneras, y con frecuencia de manera un tanto desprolija. Se ha asociado con mayor frecuencia a actividades de marketing en empresas pequeñas y con recursos limitados, por lo que depende de tácticas de marketing creativas $y$, a menudo, poco sofisticadas que hacen un gran uso de las redes personales. Por otra parte, el término se ha empleado para describir las acciones de marketing visionarias no planificadas y no lineales del empresario.

El marketing empresarial (ME) se considera como un ente integrador para abordar las actividades de marketing bajo ciertas condiciones. EI ME sintetiza los aspectos críticos del marketing y el espíritu empresarial en una conceptualización integral donde el marketing se convierte en un 
instrumento que las empresas pueden utilizar para actuar con espíritu empresarial. Esta conceptualización pretende ser un enfoque eficaz para la comercialización en una era de turbulencia y en un momento en el que las empresas enfrentan presiones únicas para mejorar la productividad de los recursos (Morris et al., 2002).

Harding (2006) propone como una posibilidad muy factible que el emprendimiento social alcance las repercusiones e impacto del emprendimiento social, con implicaciones de primer nivel en segmentos como la creación de nuevas industrias, validación de nuevos modelos de negocios y la redirección y captación de recursos para la atención de problemas sociales.

\section{Conclusiones}

El marketing del siglo XXI ha potenciado su enfoque en actividades de carácter social y/o no lucrativo, estableciendo una secuencia horizontal que abarca la expansión del marketing desde las organizaciones con objetivos lucrativos hacia las no lucrativas.
El $90 \%$ de los emprendimientos no llega a los 3 años de vida, por lo tanto, a través de la investigación analítica, prospección estratégica y la inteligencia de negocios el marketing estratégico cuenta con herramientas especializadas y metodologías de vanguardia para generar la visibilidad deseada tanto en un proyecto de emprendimiento comercial como social.

\section{Bibliografía}

Chang, Y. T., Yu, H., \& Lu, H. P. (2015).

Persuasive messages, popularity cohesion, and message diffusion in social media marketing. Journal of Business Research, 68(4), 777-782.

Doi: 10.1016/j.jbusres.2014.11.0 27

Cravens, D. (2006). Strategic marketing's global challenges and opportunities. Handbook of Business Strategy, 7(1), 63-70.

Doi: $10.1108 / 107757306106186$ 39

Dacin, P. A., Dacin, M. T., \& Matear, M. (2010). Social entrepreneurship: Why we 
don't need a new theory and how we move forward from here. Academy of management perspectives, 24(3), 37-57. https://doi.org/10.5465/amp. 24.3.37

Harding, R. (2006). Social entrepreneurship monitor. London: Global Entrepreneurship Monitor

Hunt, S. D. (1976). The nature and scope of marketing. Journal of Marketing, 40(3), 17-28. DOI: 10.1177/0022242976040003 04

Knight, G. (2000). Entrepreneurship and marketing strategy: The SME under globalization. Journal of international marketing, 8(2), 12-32. Doi: 10.1509/jimk.8.2.12.19620

Kotler, P., \& Levy, S. J. (1969). Broadening the concept of marketing. Journal of marketing, 33(1), 10-15. DOI: 10.1177/0022242969033001 03

Kotler, P., \& Zaltman, G. (1997). Social marketing: An approach to planned social change. Social Marketing Quarterly, 3(3-4), 7-20. DOI: $10.1177 / 1524500497003003$ 01

Kotler, P., \& Zaltman, G. (1971). Social marketing: an approach to planned social change. Journal of marketing, 35(3), 3-12. DOI: $10.1177 / 0022242971035003$ 02

Kotler, P. (2001). A Framework for Marketing Management. Englewood Cliffs, N.J., Prentice Hall.

Kumar, A., Bezawada, R., Rishika, R., Janakiraman, R., \& Kannan, P. K. (2016). From social to sale: The effects of firm-generated content in social media on customer behavior. Journal of Marketing, 80(1), 7-25.

Lazer, W. (1969). Marketing's changing social relationships. Journal of Marketing, 33(1), 3-9. DOI: $10.1177 / 002224296903300$ 102 
Morris, M. H., Schindehutte, M., \& LaForge, R. W. (2002). Entrepreneurial marketing: a construct for integrating emerging entrepreneurship and marketing perspectives. Journal of marketing theory and practice, 10(4), 1-19.

Doi:

10.1080/10696679.2002.11

501922

Santos, F. M. (2012). A positive theory of social entrepreneurship. Journal of business ethics, 111(3), 335351.
Seelos, C., \& Mair, J. (2005). Social entrepreneurship: Creating new business models to serve the poor. Business horizons, 48(3), 241-246. https://doi.org/10.1016/j.bus hor.2004.11.006 